\title{
LA PLACE DE LA DISCIPLINARISATION DANS L'IMAGINAIRE DE LA LANGUE ANGLAISE DANS LES ÉTABLISSEMENTS SCOLAIRES PUBLICS AU BRÉSIL
}

\author{
THE PLACE OF DISCIPLINARIZATION IN THE \\ IMAGINAIRE OF ENGLISH LANGUAGE AT PUBLIC \\ EDUCATIONAL ESTABLISHMENT IN BRAZIL
}

\author{
Elaine Pereira Daróz \\ Université Fédérale Fluminense/CAPES, Rio de Janeiro, RJ, Brasil
}

Résumé: Inscrits dans l'interface entre l'Analyse du Discours (Pêcheux) et l'Histoire des Idées Linguistiques, nous proposons dans cet article une réflexion sur la place de la disciplinarisation de la langue anglaise. L'insertion de la langue anglaise dans les écoles brésiliennes repose sur une relation hiérarchique dans la langue maternelle. Nous ferons une analyse discursive des lois qui gèrent l'éducation au pays pour mieux comprendre ce processus et ses effets dans l'imaginaire des élèves des écoles publiques au Brésil.

Mots-clés: disciplinarisation; langue anglaise; l'Analyse de discours ; l'Histoire des Idées Linguistiques.

Abstract: Inserted in the interface between Discourse Analysis (Pêcheux) and the History of Linguistic Ideas, we propose in this paper a reflection about the place of the disciplinarization of the English language in the imaginary of the students of public schools. The insertion of English language in the Brazilian schools is based on a hierarchical relationship face on the mother language. For thus, we proceeded an analysis on the laws which manage the education in this country to better understand this process.

Keywords: disciplinarization; English language; discourse analysis; history of linguistic idea.

\footnotetext{
1 Ce travail se lie à ma thèse de doctorat sur le processus de subjectivation entre le sujet et la langue, dans ce cas particulier celle des étudiants des établissements publics du Brésil et la langue anglaise, tenant en compte mes études durant mon stage de supervision doctorale à l'Université Sorbonne Nouvelle (Paris III) guidé par M. Puech, comme partie de mon travail de recherche à l'Université Fédérale Fluminense (Brésil) guidé par Professeur Silmara Dela Silva et soutenu par l'organisation brésilienne CAPES.
} 


\section{Introduction}

L'insertion de la langue anglaise dans l'institution scolaire au Brésil a eu un effet d'évidence dans la relation du sujet et la langue étrangère et, partant, dans les processus de subjectivation du sujet à la langue. Dans ce contexte, la langue anglaise est fréquemment l'unique possibilité d'apprentissage d'une langue étrangère dans l'établissement éducatif public au Brésil.

Vu que l'école occupe une place très importante dans la régularisation d'une mémoire sur la langue, nous proposons ici une réflexion sur la place de la disciplinarisation de la langue anglaise dans les établissements scolaires publics au Brésil auprès des collégiens et ses implications dans l'imaginaire du sujet par rapport à cette langue étrangère régularisé dans notre formation sociale. Dans ce travail, je présenterai des strates du discours sur la langue anglaise dans les lois que gèrent l'éducation nationale, tenant compte des conditions sociales, historiques et idéologiques dans lesquelles ces discours sont produits.

Pour cela, l'apport théorique de l'Analyse du Discours - fondée par Michel Pêcheux (1969) et développé au Brésil par Eni Orlandi - dans l'interface avec l'Histoire des Idées Linguistiques, soutient notre travail de recherche, tenant en compte la conception d'un sujet comme effet de langage, considérant la non-transparence du langage, la question de l'historicité et ses effets sur la production et la transmission du savoir dans la dimension sociale, en vue des conditions de production de ces discours nous fournissent des éléments pour notre geste d'analyse.

Dans la perspective discursive, la langue n'est ni homogène ni transparente, puisque les sens sont historiquement inscrits considérant les conditions de production du discours, c'est-à-dire du rapport entre les sujets et les situations socio-communicatives. En ce qui concerne le processus d'enseignement et apprentissage de la langue, il n'est simple ni évident, vu que le sujet est soumis d'une certaine façon à cette langue et aux conditions dans lesquelles celle-ci est transmise, ainsi que de la politique de langues est déterminée par des questions idéologiques intrinsèques au processus.

Au Brésil, la politique de langues couvre les aspects de la disciplinarisation de la langue anglaise dans notre formation sociale, ainsi l'état régule les sens sur cette langue dans le pays, ayant des conséquences dans la relation que s'établit entre le sujet et la langue, selon ce que nous pouvons observer ensuite. 


\section{La question de la disciplinarisation de la langue anglaise: une perspective analytique-discursive des lois de l'éducation nationale}

Selon Auroux (2009), l'institutionnalisation du savoir permet différents modes de signification dans et de la langue car la formalize et légitime les sens qui y sont inscrits.

A considérer les aspects historiques de l'urgence de diffusion et régulation de la langue anglaise, celle-ci est devenue une question d'état, qui fournit leur réglementation et normalisation sur les lois. Dans l'article 26 paragraphe 5 de la loi qui gère l'éducation nationale au pays - Lei de diretrizes e bases da Educação Nacional (desormais LDBEN) - assure que

Parmi les divers cours du programme scolaire sera integré, dès la cinquième année, l'enseignement d'au moins une langue étrangère moderne, dont le choix doit être pris en charge par la Communauté Scolaire, dans les possibilités de linstitution ${ }^{2}$ (notre traduction).

Bien que le document cité désigne l'enseignement obligatoire d'une langue étrangère moderne, la langue anglaise est fréquemment proposée comme la seule option d'une langue étrangère dans le programme scolaire.

Le statut d'une langue étrangère par rapport à l'enseignment de l'anglais est souvent reproduit dans le pays sous un effet d'évidence. Appuyée sur l'Analyse du discours, nous comprenons que les sens ne sont pas fixes dans la langue a priori, mais ils sont établis par une dispute idéologique afin de se régulariser dans la fomation sociale à partir d'une dénaturation des sens.

Sur la base des études de Guimarães (2007), nous proposons une dénaturation de ce term. Selon leurs études sur la politique de langues au Brésil, Guimaráes affirme que la question de la langue étrangère implique dans ses rapport avec la langue maternelle, vue qu'elle est souvent marquée par une distribution/division des espaces d'énonciation entre langues et peuples, intrinsèquement liée à la notion de civilisation. Pour l'auteur, cette relation produit effets dans les politiques de langues adoptées dans chaque état/Nation, tenant compte l'hiérarchisation d'une langue sur les autres.

Concernant le terme discipline, Chiss et Puech (2010) font la

2 "na parte diversificada do currículo será incluído, obrigatoriamente, a partir da quinta série, o ensino de pelo menos uma língua estrangeira moderna, cuja escolha ficará a cargo da comunidade escolar, dentro das possibilidades da instituição" (BRASIL, 1996). 
distinction entre discipline et la disciplinarisation, mettant en jeu la tension constitutive par laquelle les sens sont stabilisés dans la formation sociale. Selon les auteurs, dans la notion de discipline la question de transmission des connaissances se présente comme centrale, tandis que la disciplinarisation couvre les aspects pratiques de l'organisation de la transmission des connaissances ainsi que la question de l'historicité dans laquelle cette organisation est regularisée. Ainsi, la connaissance des conditions historiques et idéologiques qui finissent par établir la langue Anglaise comme "une discipline" au Brésil est essentielle pour comprendre la question posée dans ce travail.

Concernant l'enseignement d'une langue étrangère au Brésil, les Parmètres Curriculaires Nationaux (désormais PCN - Paramètres des Programmes educatifs nationaux) (BRASIL, 1999, p. 22) disent que,

Les facteurs historiques sont directment liés au rôle q'une langue spécifique représente à certains moments de l'histoire de l'humanité, [...] fréquemment, la relevance est déterminée par le rôle hégémonique de cette langue dans les échanges internationaux, gérant des implications sur les échanges dans les domaines de la culture, de l'éducation, la science, le travail, etc [...] Le cas typique est le rôle représenté par l'anglais, en fonction du poids et influence de l'économie nord-méricaine (notre traduction).

Ainsi, l'anglais est intégré d'urgence dans le programme scolaire brésilien à partir des années 1990, tenant compte de l'évènement la mondialisation afin de répondre aux exigences de la société contemporaine, suíte à la démocratisation des ordinateurs et à la facilitation de l'accès à internet.

Le document cité - PCN - a par objectif principal d'orienter les enseignants sur les questions méthodologiques et évaluatives, constituant une base à partir de laquelle les connaissances sont reproduites dans l'établissement scolaire. Les établissements scolaires, à leur tour, produisent un savoir sur la langue, régularisant les sens sur et pour cette langue, intrinsèquement guidés à la relation imaginaire constitutive qui se crée entre sujet et langue.

3 "Os fatores históricos estão relacionados ao papel que uma língua específica representa em certos momentos da história da humanidade, [...] A relevância é freqüentemente determinada pelo papel hegemônico dessa língua nas trocas internacionais, gerando implicaçóes para as trocas internacionais nos campos da cultura, da educaçáo, da ciência, do trabalho etc. O caso típico é o papel representado pelo inglês, em funçăo do poder e da influência da economia norte-americana” (BRASIL, 1999, p.22). 
Considérant le rapport des forces qui s'établit dans et par la langue, j'en déduit que l'insertion de la langue anglaise dans les établissements scolaires brésiliens, guidée sous les conditions économiques et comerciales dans le monde actuel, permet la hiérarchisation de la langue anglaise par rapport à la langue maternelle, promouvant l'imaginaire de supériorité de cette langue, intrinsèquement lié aux États-Unis et à sa culture.

J'en déduit également que ce rapport hiérarchisé est marqué par des processus historiques e idéologiques, permettant une mémoire de cette langue ayant des implications dans la politique des langues et, par conséquent, dans les modes de subjectivation qui s'établissent dans la relation entre le sujet et la langue.

Létablissement scolaire est fréquemment signifié comme un espace ouvert à la contribution des enseignants, communauté, des élèves et de leur famille, afin de promouvoir une réflexion critique en vue d'une meilleure formation du sujet. Dans ce contexte, les disciplines sont structurées à partir de connaissances prédeterminées tenant en compte les conditions sociohistoriques dans lesquelles se trouvent le pays et la communauté. Ainsi, sont présentés l'Histoire, la Géographie, la langue Portugaise et les autres disciplines disponibles dans le programme scolaire em vue d'une quantité minimun (ou maximun) de classes pour semaine/mois pour chaque discipline, ainsi que la distribution du temps de classe dans le cadre horaire (ensemble ou pas) au détriment d'autres disciplines.

En ce qui concerne la langue anglaise, contrairement à ce que l'on pourrai penser sur le enseignement de celle-ci et sur le caractère d'urgence, ce processus d'enseignement et d'apprentissage est guidé par une offre minimale de la transmission des connaissances, selon l'article 4, item III de la LDBEN (BRASIL, 1996). La loi nous dit que

le devoir de l'État comme éducateur scolaire publique sera effectivé par la garantie que des normes minimales de qualité d'enseignement, définies par la quantité minimale et la diversité, par élève, des entrées essentielles au développement du processus d'enseignement-apprentissage ${ }^{4}$ (notre traduction).

Comme nous pouvons le constater, suite au discours d'urgence à

4 "o dever do Estado com educaçấo escolar pública será efetivado mediante a garantia de [...] padróes mínimos de qualidade de ensino, definidos como a variedade e quantidade mínimas, por aluno, de insumos indispensáveis ao desenvolvimento do processo de ensinoaprendizagem" (BRASIL, 1996). 
l'enseignement de l'anglais qui marque la loi de l'éducation, le document juridique cité prend une offre minimale, "un standard minimun" d'enseignement de l'anglais dans les écoles.

Ce standard de l'enseignement d'anglais dans les établissements publics est traduit, en particulier, par des classes de courte durée, un manuel didactique souvent décontextualisé, et les salles de classes nombreuses d'environ 40 élèves. De plus, bien que les discours d'urgence d'apprentissage de la langue anglaise aux établissements scolaires, il y a un plus large lag dans le grille d'heures de classes de cette discipline compare à d'autres, considerées plus importante dans le programme éducationnel.

En fonctionnant comme un pouvoir régulateur de les sens sur et pour la langue au pays, l'état régissant aussi la stabilisation de la mémoire sur cette langue à partir de manuels, grammaires, dictionaires et ses lois.

Selon Chiss et Puech (1999), le savoir produit et disciplinarisé, circule dans les conditions historique-énonciatives spécifiques, et est guidé par une représentation disciplinaire qui a lieu dans ses processus de constitution (du savoir), réalisé dans l'intérieur des connaissances académiques, et une intégration de ces connaissances dans un complexe de connaissances qui se matérialisent dans les manuels didactiques et des actes énonciatifs, pratiques et objectifs dans l'environnement scolaire, avec des implications dans la relation de l'enseignement et de l'apprentissage.

De ce point de vue, le jeu imaginaire est constitutif de ce processus et est intrinsèque à la transmission des connaissances, tenant compte le processus de la manuélisation (PUECH, 2000) et grammatisation (FOURNIER, 2014) de la langue, aussi bien que les actes énonciatifs par ceux qui sont responsables de ce processus, comme les enseignants, les directeurs, comité de gestion scolaire et aussi les élèves.

Soutenu dans l'institution du savoir (AUROUX, 2009), l'établissements d'enseignement développé dans le processus enseignement/ apprentissage agissent pour légitimer les sens mis en circulation à partir de une reproduction, soit par le discours pédagogique des enseignants, d'équipes pédagogiques et d'autres agents de la connaissance, soit par les documents juridiques. Concomitant avec un niveau minimum de qualité de l'enseignement de l'anglais, la pratique pédagogique concernant la transmission du savoir, est donc guidée par des méthodes qui se présentent comme "plus pratiques" étant souvent décontextualisés, un geste surdéterminé par une politique de langue qui guide non seulement le savoir, mais régularise aussi une mémoire de la langue et du sujet. 
Les sens sur et par la langue anglaise régularisés dans la formation sociale

Sous l'effet de l'objectivité et de la neutralité des sens relativement stabilisés, les lois de l'éducation agissent comme un espace réglementaire d'une mémoire de la langue et son enseignement, en régularisant une mémoire sur la langue supposée homogène et une culture singularisée, dite universelle, qui est validée par les acteurs impliqués dans ce processus à partir des outils linguistiques d'appui. Comme déjà dit, nous comprenons que la reproduction de ces sens permet un imaginaire de la langue et du sujet avec des implications dans les pratiques sociales.

Par consequénce, l'anglais occupe souvent une place très importante dans notre formation sociale. Présent dans les sports, l'informatique, il est consideré la langue standard dans les conventions mondiales dans le monde corporatif et/ou académique. De même, plusieurs mots en anglais sont marqués dans notre vie quotidienne sous l'imaginaire d'une langue élitiste.

Dans l'établissement scolaire public, à son tour, les fêtes comme Halloween sont célébrées sans aucune perspective réflexive des sens qui y sont inscrits. Nous comprenons que cette pratique sociale concerne au fait à la transmission et diffusion d'une culture dit moundialisée, qui est marquée pour la simple reproduction des sens, visant à une naturalisation de cette langue et sa culture dans notre formation sociale. À mon avis, c'est l'idéologie qui sous-tend ces discours, permettant un effet de homogénéisation du sens et des sujets sous l'illusion de la objectivité du langage.

Selon Pêcheux (1999, p. 33), c'est le rôle de l'idéologie de dissimuler son fonctionnement sous le spectre de la vérité absolue, répondant au besoin du sujet pragmatique d'un «monde sémantiquement stabilisé». Sous un effet idéologique, ces sens historiquement régulées au sein de la formation sociale constituent une illusion de vérité, qui se présente sous le spectre d'une transparence du langage, comme résultant de l'interpellation de l'individu en sujet (ALTHUSSER, 1970).

Étroitement liés aux transformations sociales, les discours sur une culture universelle permettant un effacement de la contradiction qui s'établie dans le rapport de forces dans la relation entre sujets, en exposant le jeu du pouvoir inhérent à toute langue. Dans cette perspective, l'établissement educatif est un important appareil idéologique de l'état vu que, selon Althusser (1970), il'est responsable pour des enfant depuis le jeune âge, à leur inculquer les connaissances inhérentes à l'idéologie dominante. 
Sous un effet d'objectivité et neutralité des sens, les documents qui gèrent l'éducation nationale au pays agissent afin d'assurer de nouvelles pratiques sociales et, surtout, garantir l'établissement d'une mémoire sur l'objet. Ainsi, pour une légitimation des sens, les documents officiels agissant comme une mémoire institutionnalisée sur la langue anglaise et son l'enseignement dans la formation sociale, avec des implications sur la relation constitutive entre le sujet et la langue, et plus particulièrement dans l'illusion d'un passage sans entraves entre la langue matternelle et la langue étrangère. Nous pouvons donc déduire que cette illusion permet un effacement du processus complexe d'inscription du sujet à la langue 5 .

En vue du standard minimum d'enseignement de la langue anglaise assurée pour les lois que gèrent l'éducation nationale brésilienne, il y a beaucoup des cours privés d'enseignement de la langue anglaise afin de répondre aux besoins de la nouvelle societé moundialisée. Sous un effet d'évidence du sens qui se marquent dans le document officiel, l'apprentissage de la langue anglaise est régulée dans le sens d'une langue comme l'objet de consommation au lieu d'un objet du savoir.

Selon Auroux (2009, p. 43), l'instrumentalisation de la langue concerne un processus de pédagogie linguistique favorisé par la création et développement de grammaires, dictionnaires et manuels scolaires parmi d'autres outils linguistiques impliqués dans ce processus, permettant la régularisation des sens sur et par la langue à partir d'une représentation. Sous une commande apparemment anonyme de l'état, les sens sur et par la langue anglaise couvrent les sens idéologiquement surdéterminés du pouvoir économique, légitimé pour l'institution scolaires sous les formes des lois que gèrent l'éducation au pays.

À mon avis, ils sont liés aux intérêts d'une société capitaliste afin de répondre les besoins du marché des investissements. Dans cette perspective de la langue, les cours privés sont compris comme le lieu d'apprentissage de cette langue au détriment des établissements scolaires. Ceux qui régularisant un mémoire d'une langue élitiste, superposée face à l'autres, avec son implications dans l'imaginaire de cette langue pour les étudiants, et plus

\footnotetext{
5 L'usage de terme inscription au lieu d'apprentissage du sujet à la langue est appuyé dans la perspective discursive, considérant que la relation constitutif entre le sujet et la langue est marquée par un processus de soumission du sujet au les sens qui son inscrits dans cette langue, concernant les aspects socio-historiques et idéologiques de leur formation. Ainsi, le sujet a besoin de s'inscrire dans ce contexte énonciatif à partir d'une position surdeterminée par rapport à la langue maternelle. Dans ce point du vue, le terme apprentissage permet un effacement de ce processus complexe dans cette relation.
} 
particulièrement les étudiants des établissements publiques au Brésil.

Appuyés dans les sens qui régularisent ces discours sur la langue anglaise au Brésil, plus particulièrement dans l'environnement scolaire, les étudiants reproduisent cela à partir d'une mémoire du dire en vue d'une impossibilité d'apprentissage de cette langue, sachant qui la plupart vivent dans la précarité et n'ont quasiment pas accès à d'autres formes de culture.

\section{En conclusion}

La systématisation et la grammatisation de la langue anglaise ont favorisé les effets de sens pour la langue à partir d'une représentation qui, matérialisée dans les documents juridiques générateurs de l'éducation nationale, pénètrent l'environnement scolaire surdéterminant à la fois les sujets inscrits dans cet espace énonciatif, ainsi que les sens qui y sont mis en circulation, matérialisés dans les pratiques de et par la langue.

Par l'institutionnalisation des connaissances, les établissements scolaires produisent un savoir sur la langue anglaise concernant sur la base socio-historiques-idéologiques spécifique, au detriment d'expérience avec la langue en apprentissage. Sous le statut de langue étrangère, les écoles brésiliennes reproduisent une représentation de la langue et de la societé guidée par une dispute de pouvoir qui se marque dans et par la langue en tenant compte tenu une hiérarchisation de la langue anglaise par la culture nord-américaine, au detriment de la langue et culture nationale, en vue d'une mémoire sur la langue et le sujet validée par les acteurs impliqués dans ce processus à partir des outils linguistiques d'appui.

Contrairement à la disciplinarisation des connaissances en vue de transformer la réalité dans le pays, ce processus reproduit une représentation de la langue anglaise à partir d'un étatisation de celle-ci appuyée sur la mémoire du dire, relativement stabilisée dans notre formation sociale, produisant un imaginaire de langue inaccessible aux étudiants des établissements scolaires publics au Brésil.

Comme possibles conséquences d'un enseignement de bas niveau de la langue anglaise dans les établissements scolaires publics brésiliens, les étudiants ne se soucient pas de cet apprentissage dans l'environnement scolaire. Appuyés sur la mémoire du dire sur l'apprentissage de cette langue faible au pays, et tenant en compte qu'ils vivent dans une précarité de conditions économiques et, ainsi, sans accès aux cours privés d'enseignement 
de la langue, ils restent éloignés des possibilités inhérentes à la nouvelle société du savoir.

\section{Références}

ALTHUSSER, L. Ideologia e Aparelhos ideológicos do Estado. Lisboa: Presença, 1970.

AUROUX, S. A questáo da origem das línguas, seguido de Historicidade das ciências. Campinas, 2008.

A revoluçáo tecnológica da gramatizaçáo. Campinas: Editora da Unicamp, 2009.

Listas de palavras, dicionários e enciclopédias: o que nos ensinam os enciclopedistas sobre a natureza dos instrumentos linguísticos. Língua e Instrumentos Linguísticos, Campinas, n. 20, 2008.

BRASIL. Lei 9.394 - Lei de diretrizes e bases da educaçáo nacional. Brasília: Imprensa Nacional. 1996.

Parâmetros curriculares nacionais terceiro e quarto ciclos do ensino fundamental: língua estrangeira. Brasília: MEC/SEF, 1998.

- Parâmetros curriculares nacionais do ensino médio: língua estrangeira. Brasília: MEC/SEF, 2000.

CHISS, J-L; PUECH, C. Entrevista realizada durante o II CIAD, evento ocorrido na UFSCAR, São Carlos, em 2009. In: Linguagem, Teoria, História. Série Cogitare, Santa Maria, n.11, 2010.

FOURNIER, J-M; RABY, V. Retour sur la grammatisation: l'extension de la grammaire latine et la description des langues vulgaires. - Histoire 
des Théories Linguistiques - UMR 7597. In: ARCHAIMBAULT, S; FOURNIER, J-M; RABY, V. Penser l'histoire des savoirs linguistiques. Hommage à Sylvain Auroux. ENS Editions, p. 337-350, 2014.

GUIMARÃES, E. R. J. Política de línguas na linguística brasileira. In: ORLANDI, E. P. (Org). Política linguística no Brasil. Campinas: Pontes, 2007.

PÊCHEUX, M. Análise automática do discurso. In: GADET, F.; HAK, T. (Org.). Por uma análise automática do discurso: uma introdução à obra de Michel Pêcheux. Campinas: Editora da Unicamp, [1969] 2010.

Papel da memória. In: ACHARD, P. (org.). Papel da memória. Campinas: Pontes, [1983] 1999.

PUECH, C. Manuélisation et disciplinarisation des savoirs de la langue. Les carnets du Cediscor, Paris, n.5, p.15-30, 2000. Disponível em http:// journals.openedition.org/cediscor/267. Acesso em 5 out. 2018.

DOI - http://dx.doi.org/10.5902/2179219436588 